\title{
Evolução Arquitetural em Sistemas de Controle de Satélites
}

\author{
Adair J. Rohling ${ }^{1}$, Valdemar V. G. Neto ${ }^{2}$, Elisa Y. Nakagawa ${ }^{3}$, Mauricio G. V. Ferreira ${ }^{4}$ \\ ${ }^{1}$ Universidade Tecnológica Federal do Paraná (UTFPR) \\ Av. Prof ${ }^{a}$ Laura Pacheco de Bastos, 800 -CEP 85053-525. Guarapuava- PR - Brazil \\ ${ }^{2}$ Universidade Federal de Goiás (UFG) \\ CEP 74690-900 Goiânia - Goiás - Brasil \\ ${ }^{3}$ Universaidade de São Paulo (USP) - ICMC \\ CEP: 13566-590 - São Carlos - SP - Brasil \\ ${ }^{4}$ Instituto Nacional de Pesquisas Espaciais (INPE) \\ Av. dos Astronautas, 1758 - CEP 12227-010. São José dos Campos - SP - Brasil
}

\{adairrohling,vgracianoneto\}@gmail.com, elisalicmc.usp.br, mauricio@ccs.inpe.br

\begin{abstract}
Satellite Control Systems (SCS) are responsible for maintaining the proper functioning of orbiting satellites. These systems are constantly evolving architecturally to meet interoperability requirements and technological advances in satellites. The Satellite Control Center (CCS) of the National Institute for Space Research (INPE) has been proposing and developing software solutions for this domain since 1980. Thus, the purpose of this report is to present the history of the architectural and technological evolution of the SCS developed by CCS. The architectural advance in this domain has evolved to the establishment of a Reference Architecture for Satellite Control Systems, which has shown benefits of architectural reuse and reduced efforts during the development and integration phases.
\end{abstract}

Resumo. Sistemas de Controle de Satélites (SCS) são responsáveis por manter o funcionando adequado dos satélites em órbita. Esses sistemas estão em constante evolução arquitetural para atender requisitos de interoperabilidade e os avanços tecnológicos dos satélites. O Centro de Controle de Satélites (CCS) do Instituto Nacional de Pesquisas Espaciais (INPE) vem propondo e desenvolvendo soluções de softwares para este domíno desde 1980. Assim, o objetivo deste relato é apresentar o histórico da evolução arquitetural e tecnológica dos SCS desenvolvidos pelo CCS. O avanço arquitetural neste domínio tem evoluído para o estabelecimento de um Arquitetura de Referência para Sistemas de Controle de Satélites, a qual tem apresentado benefícios do reúso arquitetural e a redução de esforços durante as fases de desenvolvimento e integração.

\section{Sistemas de Controle de Satélites}

O controle de satélites em órbita está associado às atividades de controle da atitude, controle da órbita, controle da plataforma de serviço e carga útil. O controle de satélites é realizado por estações terrenas e pelos Centros de Controle de Satélites (CCS). Os CCS contém todos os equipamentos, simuladores, serviços e sistemas para apoiar a realização das atividades de controle de satélites durante o seu ciclo de vida no espaço e também durante o planejamento e execução da missões. 
O INPE possui seu CCS localizado em São José dos Campos(SP) e as estações terrenas remotas estão localizadas em Cuiabá (MT) e Alcântara (MA). Todas as ações de controle dos satélites são planejadas, coordenadas e executadas a partir do CCS e a comunicação em solo com os satélites em órbita são realizadas a partir das estações terrenas. Alguns satélites desenvolvidos pelo INPE são: SCD1, SCD2, SACI 1, SACI2, NANOSATC-BR1, NANOSATC-BR2, UbatubaSat, CBERS-1, CBERS-2, CBERS-2B, CBERS-3, CBERS-4 e CBERS-4A e Amazonia-1.

Soluções em software para SCS são complexas e executam inumeras funções, tais como: receber, processar e armazenar as telemetrias, realizar cálculo de manobras, gerar, validar e enviar os telecomandos às estações, realizar configuração operacional da carga útil, determinar e propagar as órbitas e a atitude dos satélites, receber e processar medidas de distância de velocidade e angulares, preparar e realizar manobras com os satélites, gerar as previsões de passagem dos satélites pelas estações e gerar planos de voo para os satélites controlados. Além dessas funcionalidades, tais soluções devem atender requisitos de escalabilidade para suportar ao aumento significativo do número de satélites controlados e requisitos de intereporabilidade para permitir o compartilhamento de recursos de infraestrutura e de operações entre diversas organizações espaciais.

\section{Evolução Arquitetural dos Softwares para SCS}

Em paralelo ao contexto dos SCS, arquiteturas de software formam a base para todo o sistema complexo de software bem-sucedido, desempenhando um papel importante na determinação da qualidade de sistemas. Assim, o CCS vem desenvolvendo e evoluindo sua arquitetura para os SCS de forma que atendam aos novos requisitos deste domínio e seus avanços tecnológicos. Na sequência é apresentado um breve resumo das principais arquiteturas dos SCS desenvolvidas pelo CCS.

\subsection{Arquitetura Centralizada Baseada em Mainframes}

O primeiro SCS foi desenvolvido na década de 80 com o surgimento da Missão Espacial Completa Brasileira (MECB) ${ }^{1}$. O sistema possuía uma arquitetura centralizada baseada em mainframes, a linguagem Fortram foi utilizada em sua implementação com aproximadamente 155 mil linhas de código. Este sistema era composto pelo softwares operacionais de controle de satélites e estações terrenas. Este sistema foi desenvolvido especialmente para controlar satélites desenvolvidos pelo INPE, em especial os satélites SCD1 e SCD2.

\subsection{Arquitetura Cliente-Servidor}

Um nova versão do SCS foi desenvolvida para ser utilizada no controle do primeiro satélite brasileiro de sensoriamento remoto (CBERS). Esta versão apresentava uma arquitetura cliente-servidor disponível em plataformas PCs e o uso de banco de dados relacional. O desenvolvimento foi baseado no paradigma orientado a objetos e $\mathrm{C}++$ foi a linguagem de programação utilizada.

\subsection{Arquitetura Baseada em Objetos Distribuídos}

Outra versão arquitetural para SCS é apresentada em [Ferreira 2001]. Esta arquitetura é baseada em objetos distribuídos e o uso de middleware aderente especificação CORBA.

\footnotetext{
${ }^{1}$ http://www.aeb.gov.br/timelinr/1979
} 
Foi utilizado a linguagem Java durante o desenvolvimento com aproximadamente 9 mil linhas de código. Está arquitetura compreende os objetos distribuídos do domínio e os serviços de agentes, persistência, segurança e balanceamento. Esta arquitetura apresenta inúmeras vantagens em relação aos sistemas centralizados: melhor escalabilidade, confiabilidade, desempenho e a busca por uma arquitetura aberta e heterogênea.

\subsection{Framework SATCS}

Outro avanço na arquitetura dos SCS no INPE foi a criação e uso do framework SATCS [Cardoso 2008]. O projeto do SATCS está baseado na filosofia de desenvolvimento de software orientado a componentes e serviços. O framework fornece, sem impacto na arquitetura original, um conjunto fundamental de serviços customizáveis aos requisitos operacionais para diferentes missões espaciais.

A arquitetura lógica do framework SATCS é composta de quatro camadas, conforme apresentado na Figura 1, que distribuem as funcionalidades de persistência e acesso de dados, serviços de domínio especializado e apresentação do usuário.

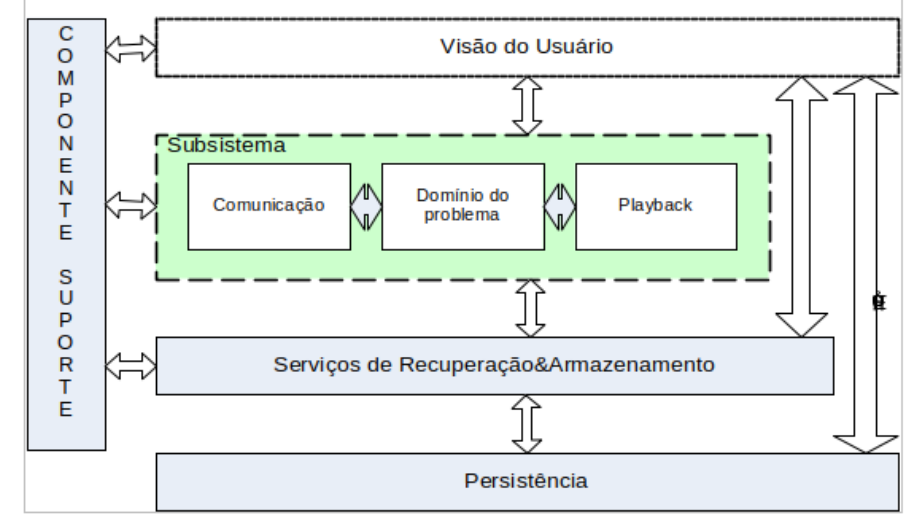

Figure 1. Arquitetura Lógica - SATCS

O framework SATCS também provê uma biblioteca de funções auxiliares que podem ser reutilizadas para: recuperar de parâmetros de configuração da base de dados operacional, armazenar e visualizar de mensagens de eventos, agendar serviços temporizados e realizar a codificação/decodificação binária de pacotes de dados a partir de metadados de operação.

\subsection{Arquitetura de Referência SCS}

A arquitetura de referência é uma abstração do conhecimento arquitetônico para um determinado domínio e assim fornece diretrizes importantes para a especificação de arquiteturas de software [Nakagawa et al. 2011]. Assim, conforme definido em [Rohling et al. 2019], o desenvolvimento de uma Arquitetura de Referência para Sistemas de Controle de Satélites (ARSCS) apresenta-se como um elemento essencial para construção e evolução arquitetural neste domínio. O objetivo da construção da ARSCS é servir como orientação para o desenvolvimento, padronização e evolução dos SCS.

Para estabelecer a ARSCS foi adotado o processo sistemático ProSA-RA, definido em [Nakagawa et al. 2014], que é constituído de quatro etapas fundamentais que guiam 
o desenvolvimento de arquiteturas de referência: investigação de fontes de informação, estabelecimento de requisitos arquiteturais, design da arquitetura e a avaliação da arquitetura de referência.

Na etapa de investigação de fontes de informação foram consultados os principais padrões internacionais de sistemas espaciais, os quais se destacaram as publicações realizadas pela European Cooperation for Space Standardization (ECSS) ${ }^{2}$ e pelo Consultative Committee for Space Data Systems (CCSDS) ${ }^{3}$. Também foram coletadas informações de operadores de estações terrestres, controladores de satélites, engenheiros de sistemas espaciais, pesquisadores e professores da área de arquitetura de referência.

$\mathrm{Na}$ etapa de estabelecimento dos requisitos foram definidos os principais requisitos arquiteturais do domínio e os requisitos arquiteturais técnicos. Na etapa de design da arquitetura foram descritas e representadas as visões arquiteturais de fluxo de dados, estrutural, composição e lógica. Na Figura 2 é apresentado a visão de fluxo de dados dos principais componentes das categorias de gerenciamento, coordenação, negócio e persistência.

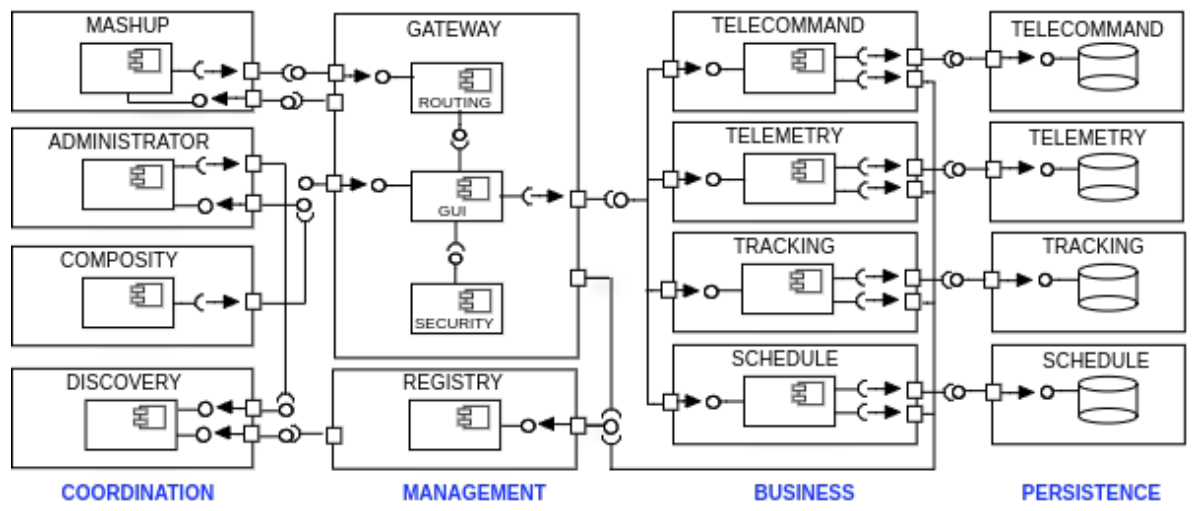

Figure 2. Visão de Fluxo de Dados - ARSCS

A avaliação da ARCSS foi realizada usando diferentes técnicas e métodos. Para verificar a coerência entre os requisitos e o design arquitetural foi realizado um mapeamento entre estes artefatos. Uma comparação foi realizada entre os requisitos da ARSCS e as funcionalidades dos principais sistemas existentes. Também foi realizada uma avaliação utilizando o modelo RAModel [Nakagawa et al. 2012], que provê uma completa lista de elementos necessário para construir uma arquitetura de referência.

\section{Aplicação da ARSCS}

Para observar os benefícios da adoção da arquitetura de referência e fornecer evidências de sua viabilidade foi desenvolvido um produto de software para controle de microssatélites, denominado MicroSatCS. A arquitetura do MicroSatCS foi instanciada em conformidade com a ARSCS.

\subsection{Visão Geral MicroSatCS}

O desenvolvido do MicroSatCS foi realizada em consequência da quantidade considerável de projetos atuais e também das perspectivas de crescimento do número de

\footnotetext{
${ }^{2}$ https://ecss.nl/

${ }^{3}$ https://public.ccsds.org/
} 
microssatélites. O processo de desenvolvimento foi baseado no ciclo definido pela ECSS [European Cooperation for Space Standardization 2004].

$\mathrm{Na}$ etapa do design arquitetural os componentes necessário para atender os requisitos foram definidos a partir da ARSCS. Na análise de definição do modelo de componente foram considerados os requisitos arquiteturais de interoperabilidade, independência e escalabilidade definidos na ARSCS. Em consequência foi definido o modelo de componente denominado CubeSoft. Sua taxonomia é apresentada na Figura 3 por meio de um modelo de features. CubeSoft é um modelo de componente descrito de forma abstrata e independente de linguagem e plataforma de execução.

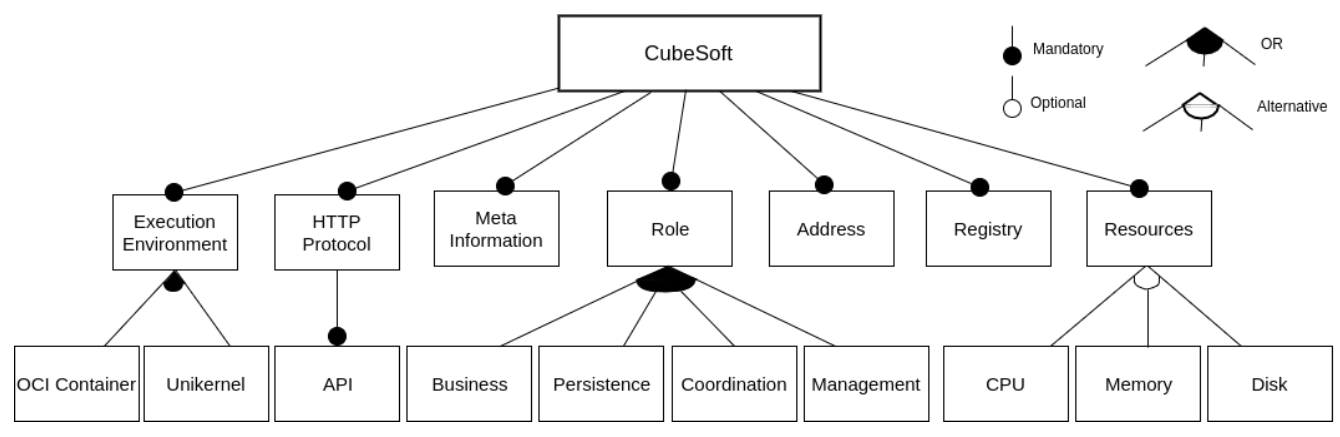

Figure 3. Taxonomia CubeSoft

Na etapa de operação do MicroSatCS é possível executar as principais funcionalidades do sistema, conforme apresentado na Figura 4, que também apresenta o sistema em operação realizando o rastreamento do satélite UbatubaSat.

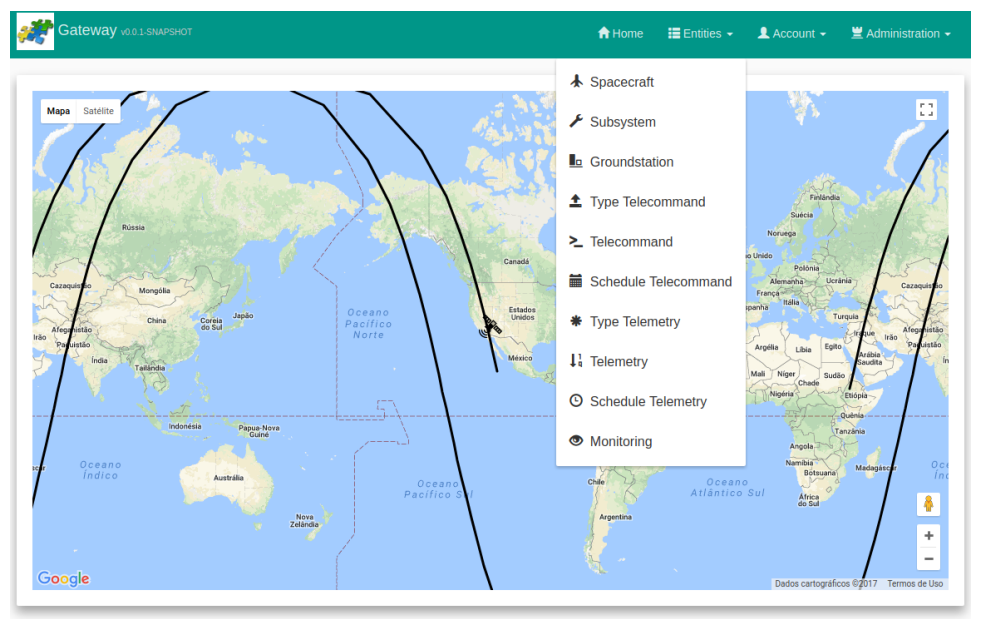

Figure 4. MicroSatCS em Operação

\section{Conclusões}

O CCS vem somando conhecimento durante várias décadas com a evolução arquitetural dos seus SCS. Sistemas com diferentes tecnologias e estilos arquiteturais foram desenvolvidos para atender este domínio que requer um alto grau de confiabilidade.

O modelo arquitetural baseado em características de generalização, como uma arquitetura de referência, possui um custo elevado de criação, porém expectativa na redução 
dos custos podem ser observadas durante a instanciação de uma arquitetura concreta. SCS podem ser facilmente construídos a partir da ARSCS, reduzindo o tempo e os esforços de retrabalho, além de melhorar a interoperabilidade e o compartilhamento de recursos terrestres entre organizações espaciais.

Resultados obtidos no desenvolvimento do MicroSatCS, em específico durante a etapa do projeto arquitetural e considerando o uso da ARSCS, apresentaram uma redução de $65 \%$ em termos de esforço de pessoa-horas. Esta análise considerou principalmente dados quantitativos ocorridos principalmente na definição dos requisitos, estilo arquitetural, padrões de projeto e na representação dos modelos arquitetônicos. Além desses resultados obtidos, destacamos a necessidade de realizar uma avaliação mais criteriosa envolvendo um maior quantitativo de sistemas desenvolvidos e integrados.

\section{References}

Cardoso, P.; Barreto, J. C. L. H. L. (2008). Using Design Patterns, Components and Metadata to Design the Command and Monitoring Frameworks of the INPE's Satellite Control System. SpaceOps Conference. https://doi.org/10.2514/6.2008-3591.

European Cooperation for Space Standardization (2004). ECSS-E-10 Part 1B - System Engineering - part 1: requirements and process.

Ferreira, M. G. V. (2001). Uma arquitetura flexível e dinâmica para objetos distribuídos aplicada ao software de controle de satélites. Instituto Nacional de Pesquisas Espaciais (INPE) - Tese de Doutorado em Computação Aplicada, http://urlib.net/rep/83LX3pFwXQZ5Jpy/wMD3P.

Nakagawa, E. Y., Antonino, P. O., and Becker, M. (2011). Reference architecture and product line architecture: a subtle but critical difference. In Proceedings..., pages 207211, Berlin, Heidelberg. European Conference on Software Architecture, 5., 2011, Springer-Verlag.

Nakagawa, E. Y., Guessi, M., Maldonado, J. C., Feitosa, D., and Oquendo, F. (2014). Consolidating a process for the design, representation, and evaluation of reference architectures. In Proceedings..., pages 143-152. Working IEEE/IFIP Conference on Software Architecture, 2014.

Nakagawa, E. Y., Oquendo, F., and Becker, M. (2012). RAModel: A reference model for reference architectures. In Proceedings..., pages 297-301. Joint Working IEEE/IFIP Conference on Software Architecture and European Conference on Software Architecture, 2012, IEEE.

Rohling, A. J., Neto, V. V. G., Ferreira, M. G. V., Dos Santos, W. A., and Nakagawa, E. Y. (2019). A reference architecture for satellite control systems. Innovations in Systems and Software Engineering, pages 1-20. 